\title{
On The Positive Correlation Between Income and Patience*
}

\author{
Rubens Penha Cysne ${ }^{\dagger}$
}

Contents:1. Introduction; 2. The Basic Model; 3. A More General Model; 4. The Long-Run Average Utility and Wage; 5. Main Results; 6. Conclusions;

Keywords: Time preference; Wage; Income; Invariant Distribution; Search. JEL Code: J30; J31.

This paper uses a standard job-search model to provide a new explanation for the empirically documented positive correlation between patience and average income: more patient workers, by rejecting less favorable wage offers, end up with a higher average income in the long run. This is not clear at first glance, since rejecting more offers also leads to more time of unemployment. It turns out that more patient workers always "in in the end". The result is proved for the case of a linear utility. When utility is not linear, a sufficiency condition is provided. Average utility always increases with patience. A counterexample is used to show that such conclusions could not follow from a direct analysis of the value function. A second example is offered at the end to illustrate the main points of the paper.

\section{INTRODUCTION}

There is a considerable amount of empirical support showing the existence of heterogenous discounting among households (see, e.g., Dreyfus and Viscusi (1995), Samwick (1998) or Warner and Pleeter (2001)) and supporting the idea that patience (willingness to defer gratification) correlates positively with different measures of economic success.

Regarding this second point, Hausman (1979), for instance, presents a model of individual behavior in the purchase and utilization of energy-using durables and shows that discount rate varies inversely with income. Lawrance (1991) estimated consumption Euler equations using the Panel Study of Income Dynamics and showed that subjective rates of time preferences can be up to 6 percentage points

*I am thankful to participants of workshops at the Department of Economics of the University of Chicago and at the Graduate School of Economics of the Getulio Vargas Foundation for their comments.

${ }^{\dagger}$ Professor of Economics at the Graduate School of Economics of the Getulio Vargas Foundation (EPGE/FGV) and a Visiting Scholar at the Department of Economics of the University of Chicago. E-mails: rpcysne@uchicago.edu; rubens@fgv.br. 
higher in the top 5 percent of the labor-income distribution than in the bottom fifth percentile. Other references in the same line are Maital and Maital (1977) and Wärneryd (1999).

Theoretically, different models have been devised to explain the positive correlation between patience and income or wealth. The basic intuition underlying these models is that more patient individuals are likely to invest more heavily in human capital, becoming more skilled and earning higher labor and/or capital income.

An early literature based on deterministic models (e. g., Becker (1980) and Lucas and Stokey (1984)) has shown that, under additive preferences, time-preference heterogeneity can lead to degenerate wealth distributions.

A more recent trend in this literature avoids this radical result by adding different assumptions to the basic model. Among them, Andolfatto and Redekop (1998) assume that a fraction of the agents die each period, and bequeath their wealth to descendants with independently random discount parameters. This assumption allows these authors to obtain a long-run positive correlation between patience and average income without the problem of having a degenerate distribution of wealth or income. In the same line, Carroll (1997) works out an assumption of market incompleteness, whereas Krusell and Smith (1998) suppose that households face mean-reverting shocks to their time-preference parameter.

My first objective here is to show that a version of MacCall (1970) job search model, a relatively simple and well known model, can also provide a mechanism by means of which patience correlates positively with average income. ${ }^{1}$

The result is not clear at first sight, for two reasons. First, because it is based on the average wage obtained under the invariant distribution that emerges in the process of job search, rather than directly on the reservation wage, the variable which is usually the focus of job-search models.

Second, because when patience is higher, two facts lead to opposite effects regarding the average wage: more patient workers tend to have higher reservation wages and therefore higher wages; but also to reject more wage offers and to spend more time unemployed.

The first main point of this paper is showing that the final result of this trade-off is always favorable for the more patient worker when utility is linear. ${ }^{2}$ When utility is not linear, a sufficient condition is provided for the result to be true. The more patient worker always "wins in the end", meaning that she or he ends up with a higher average income, regardless of spending more time unemployed.

This conclusion, proved here, and proved by means of a counter-example not to follow directly from an analysis based solely on the value function (it is necessary to derive the invariant distribution of wages in the economy in order to formalize it), provides a new way to understand the empirically assessed positive correlation between income and patience.

Relatively to other theoretical explanations of the link between patience and measures of economic success, it is formally simpler and has the distinguishing feature of not depending upon the accumulation of capital or human capital.

A second objective of this paper is to verify how the correlation between patience and income, and between patience and average utility, varies as a function of the probability of unemployment and of the probability that unemployed workers do not get a job offer. In order to do so, I provide a small generalization of Stokey et al. (1989) version of McCall's model, which results in one additional parameter being added to the model. All calculations are developed under this more general version. The respective result for the more basic model can be obtained by considering that the new parameter is equal to zero.

The remainder of this paper is organized in the following way. Section 2 presents the basic model and advances, in its more simplified framework, some of the results to be obtained later. All developments are made using a general concave utility. Section 3 generalizes the basic model and Section 4

\footnotetext{
${ }^{1}$ The paper abstracts from capital income. All income here is labor income.

${ }^{2}$ About this assumption, see Remark 3 . The fact that average utility (rather than income) increases with patience is always true and does not depend upon this assumption.
} 
develops the calculations leading to the average utility and to the average wage in the economy. The main results are proved in Section 5, where two examples are also offered. Section 6 concludes.

\section{THE BASIC MODEL}

The basic search model used in this section to show that patience correlates positively with income is (Stokey et al., 1989, c. 10, p. 304) version of McCall (1994) model. ${ }^{3}$ The next sections generalize this model and prove the results.

The economy has a continuum of workers indexed by $[0,1]$. For all purposes, one can think of a small country in which workers receive their job offers from foreign firms.

Consider the measurable space $(\Omega, \mathcal{F}, p)$ and, in this space, the measure $\mu$ induced by the wage function $w: \Omega \rightarrow[0, \bar{w}]$ (with $0<\bar{w}<\infty)$. In the induced space $\left([0, \bar{w}], \mathcal{B}_{[0, \bar{w}]}, \mu\right)$, denote by $F(t)$ the distribution function that ( $\mu$ a.e. uniquely) determines the measure $\mu: F(t)=p(w \leq t)$. The distribution $F($.$) is assumed to satisfy 0<E w<\bar{w}$.

The (representative) worker is not allowed to borrow or to lend. His consumption $c_{t}$ is equal to his income $w_{t}$ in each period. The consumer maximizes the expected present value of the utility derived from consumption:

$$
E\left(\sum_{t=0}^{\infty} \beta^{t} U\left(c_{t}\right)\right)
$$

The utility function $U($.$) is assumed to be continuously differentiable, strictly increasing and con-$ cave $\left(U^{\prime \prime}(w) \leq 0\right)$, with $U(0)=0$ and $U^{\prime}(0)<\infty$. By assumption $0<\beta<1$.

State " $w$ " corresponds to a job offer $w$ at hand, and state " 0 " to no job offer that period. In state $w$ the worker can accept or turn down the offer. If he accepts it, by assumption he is employed with that wage, facing, each period, a probability of unemployment given by $\theta, 0<\theta<1$. If he does not accept the offer he will be this period in state 0 . Being in state zero the only thing he can do is to wait till next period for a new job offer which, in the present version of the model, happens with probability one. Wages are normalized so that the compensation wage, when unemployed, is equal to zero. While employed, the individual is not allowed to search for wage offers.

The reservation wage $w^{*}$ in this case is given by equation 10.7 .6 in (Stokey et al., 1989, p. 307).

Remark 2.1. In this economy each single worker is subject to an infinite cycle of employment, lay-off, and unemployment. The average wage (and average utility) in which we are interested here is the one of such a representative worker along his (supposedly infinite) life. Due to the law of large numbers, the average wage (average utility) also happens to be equal to the cross-sectional average of the wages (utilities) of all workers in the economy, employed and unemployed, in a single point in time.

Call the average wage $w_{A}:{ }^{4}$

\footnotetext{
${ }^{3}$ Other sources where this type of model can be found are Sargent and Ljungqvist (2002) and Sargent (1987).

${ }^{4}$ Exercise 10.8.d of Stokey et al. (1989) asks the average wage of employed workers, $w_{A}^{E}$. This is not the statistic in which we shall be interested here. In any case, as we shall se in the next section, this value can be easily calculated using the invariant cross-sectional distribution of wages in the economy, and conditioning it on the event that the worker is employed. The result is:
}

$$
w_{A}^{E}=\int_{\left[w^{*}, \bar{w}\right]} \frac{w d F(w)}{\mu(A)}
$$

It is easy to see that $w_{A}^{E}$ could not be given by (2). Take an economy in which $\mu$ assigns probability one to $w=\hat{w}$. In any cross section, the average wage of employed workers has to be equal to $\hat{w}$, a fact which follows trivially from (1), but not from (2). 


$$
w_{A}=\int_{\left[w^{*}, \bar{w}\right]} \frac{w d F(w)}{\theta+\mu(A)}
$$

We are going to see in Section 5, under a more general framework, which has the one of this section as a particular case, that $w_{A}$ is an increasing function of patience when the utility is linear.

\section{A MORE GENERAL MODEL}

In this section I present a slight generalization of the model introduced in Section 2 and use this new model to develop all calculations leading to the main results of the paper.

Suppose now that, in each period, the worker faces a probability $\alpha, 0<\alpha<1$ of getting no job offer when he is unemployed. With $v(w)$ standing for the value function when the state is "wage offer $w$ at hand", $\mathcal{A}$ for accept and $\mathcal{R}$ for reject, the recursive version of the consumer's problem is given by:

$$
v(w)=\max _{\mathcal{A}, \mathcal{R}}\{U(w)+\beta[(1-\theta) v(w)+\theta v(0)], v(0)\}
$$

where

$$
v(0)=\beta\left[(1-\alpha) \int_{[0, \bar{w}]} v(w) d F(w)+\alpha v(0)\right]
$$

Making $X=\frac{1-\alpha}{1-\alpha \beta}$ :

$$
v(0)=\beta X \int_{[0, \bar{w}]} v(w) d F(w)
$$

Note that

$$
v(0)=U\left(w^{*}\right)+\beta v(0)
$$

This follow from (3) and from the definition of the reservation wage.

Proposition 3.1 (Determination of the Reservation Wage). There is a unique $w^{*} \in[0, \bar{w}]$ such that:

$$
v(w)=\left\{\begin{array}{cc}
v(0) & \text { if } w \leq w^{*} \\
\frac{U(w)+\beta \theta v(0)}{1-\beta(1-\theta)} & \text { if } w>w^{*}
\end{array}\right\}
$$

Proof. Trivial.

Proposition 3.2. $w^{*}$ (the reservation wage) is implicitly determined by the equation:

$$
U\left(w^{*}\right)=\frac{\beta(1-\alpha)}{1-\beta(1-\theta)} \int_{\left[w^{*}, \bar{w}\right]}\left(U(w)-U\left(w^{*}\right)\right) d F(w)
$$

Proof. From (4) and (6):

$$
v(0)=X \beta \int_{\left[0, w^{*}\right]} v(0) d F\left(w^{\prime}\right)+X \beta \int_{\left[w^{*}, \bar{w}\right]} \frac{U(w)+\beta \theta v(0)}{1-\beta(1-\theta)} d F(w)
$$

Collecting terms:

$$
v(0)=\frac{X \beta}{(1-\beta)\left(1-\beta F\left(w^{*}\right) X\right)+(1-\beta X) \beta \theta} \int_{\left[w^{*}, \bar{w}\right]} U(w) d F(w)
$$


Use (6), (5) and the definition of the variable $X$ to get:

$$
U\left(w^{*}\right)=\frac{\beta(1-\alpha)}{1-\beta\left(\alpha+F\left(w^{*}\right)(1-\alpha)-\theta\right)} \int_{\left[w^{*}, \bar{w}\right]} U(w) d F(w)
$$

Next, add and subtract $\frac{\beta(1-\alpha)}{1-\beta\left(\alpha+F\left(w^{*}\right)(1-\alpha)-\theta\right)} \int_{\left[w^{*}, \bar{w}\right]} U\left(w^{*}\right) d F(w)$ to the second member of the equation above and collect terms to get (7).

Proposition 3.3. The reservation wage is an increasing function of the worker's patience $(\beta)$, a decreasing function of the probability that unemployed workers find no job offer $(\alpha)$ and a decreasing function of the probability of layoff $\theta$.

Proof. All assertions follow from straightforward applications of the implicit function theorem.

Define:

$$
G\left(w^{*}, \beta, \alpha, \theta\right)=U\left(w^{*}\right)-\frac{\beta(1-\alpha)}{1-\beta(1-\theta)} \int_{\left[w^{*}, \bar{w}\right]}\left(U(w)-U\left(w^{*}\right)\right) d F(w)
$$

and use (7) to obtain:

$$
\begin{gathered}
\frac{\partial w^{*}}{\partial \beta}=\frac{U\left(w^{*}\right)}{\beta[1-\beta(1-\theta)] U^{\prime}\left(w^{*}\right)\left[1+\frac{\beta(1-\alpha)\left(1-F\left(w^{*}\right)\right)}{1-\beta(1-\theta)}\right]}>0 \\
\frac{\partial w^{*}}{\partial \alpha}=\frac{-U\left(w^{*}\right)}{[1-\alpha] U^{\prime}\left(w^{*}\right)\left[1+\frac{\beta(1-\alpha)\left(1-F\left(w^{*}\right)\right)}{1-\beta(1-\theta)}\right]}<0 \\
\frac{\partial w^{*}}{\partial \theta}=\frac{-\beta U\left(w^{*}\right)}{[1-\beta(1-\theta)] U^{\prime}\left(w^{*}\right)\left[1+\frac{\beta(1-\alpha)\left(1-F\left(w^{*}\right)\right)}{1-\beta(1-\theta)}\right]}<0
\end{gathered}
$$

Remark 3.1. Note in (8) that the influence of $\alpha$ over $\frac{\partial w^{*}}{\partial \beta}$ cannot be directly determined from (8). The direct effect (considering the $\alpha$ which appears in the denominator of $\frac{\partial w^{*}}{\partial \beta}$ ) is positive, but the effect through the reservation wage (given 9), and taking into consideration that $U^{\prime}\left(w^{*}\right)>0$ and $U^{\prime \prime}\left(w^{*}\right)<0$ is negative.

\section{THE LONG-RUN AVERAGE UTILITY AND WAGE}

The determination of the average wage here follows, with some changes, the procedure outlined in (Stokey et al., 1989, section 10.8, p. 308).

The reservation wage $w^{*}(j)$ divides $[0, \bar{w}]$ into two regions: the acceptance region $A=\left[w^{*}, \bar{w}\right]$ and the non-acceptance region $A^{c}=\left[0, w^{*}\right]$. Note that $A$ and $A^{c}$ depend on the value of $\beta$. The rules of the optimization followed by the worker define a transition function $P:[0, \bar{w}] \times \mathcal{B}_{\bar{w}} \rightarrow[0,1]$, of wage offers.

If the current state (given by the wage offer) is $w \in A^{c}$, the probability of having an offer next period in any borelian $B \subset[0, \bar{w}]$ is given by $(1-\alpha) \mu(B)+\alpha$, if $0 \in B$, and $(1-\alpha) \mu(B)$ if $0 \notin B$. Alternatively, if the current state is $w \in A$, the worker can only lose his job (with probability $\theta$ ) or keep the same wage next period. Therefore, with probability zero he will have a wage next period in a borelian $B$ that does not contain either 0 or $w$. If the borelian $B$ contains 0 , but not $w$, or $w$ but not zero, the transition probabilities are, respectively, $\theta$ and $1-\theta$. If it contains both, since these are disjoint events (because $0 \notin A), P(w, B)=1$. 
This transition function implies the only ergodic set of the problem to be the whole (induced) sample space, $[0, \bar{w}]$. It also defines an invariant measure $\lambda$ in $[0, \bar{w}]$ of wage offers in the economy. To find the average wage of a worker along time (or the cross sectional average wage of all workers in the economy - see Remark $1-$ ), we need to find this invariant measure $\lambda$.

In order to do so, notice the following. A worker will be offered a wage in the acceptance region $A$ in period $t+1$ if either he already had this wage in $A$ in period $t$ and is not laid off or if he was unemployed, receives a wage offer, and this wage offer falls in $A$. Formally:

$$
\lambda_{t+1}(A)=\lambda_{t}(A)(1-\theta)+\lambda_{t}\left(A^{c}\right)(1-\alpha) \mu(A)
$$

Taking limits, ${ }^{5}$ this leads to the following invariant measure of the acceptance region $A(\lambda=$ $\left.\lim _{t \rightarrow \infty} \lambda_{t}\right)$ :

$$
\lambda(A)=\frac{(1-\alpha) \mu(A)}{\theta+(1-\alpha) \mu(A)}
$$

Since $A \cup A^{c}=[0, \bar{w}]$ and $A \cap A^{c}=\emptyset$ :

$$
\lambda\left(A^{c}\right)=1-\lambda(A)=\frac{\theta}{\theta+(1-\alpha) \mu(A)}
$$

Now suppose $\{0\} \subset C \subset A^{c}$. A worker will have a wage offer in this set $C$ either if she is currently employed and is laid off, if she is unemployed and gets no wage offer or if she is unemployed and gets a wage offer in $C$. Therefore:

$$
\lambda_{t+1}(C)=\lambda_{t}(A) \theta+\lambda_{t}\left(A^{c}\right)(\alpha+(1-\alpha) \mu(C))
$$

Using (12) and (13), this implies that for sets $\{0\} \subset C \subset A^{c}$ the invariant measure reads:

$$
\lambda(C)=\frac{\theta(1-\alpha) \mu(A)+\alpha \theta}{\theta+(1-\alpha) \mu(A)}+\frac{\theta(1-\alpha) \mu(C)}{\theta+(1-\alpha) \mu(A)}
$$

Finally, a worker will have a wage offer in a set $C \subset A$ in data $t+1$ if either she already has a wage $w \in C$ and keeps it, or if she is unemployed, happens to receive a wage offer, and this wage offer is in $C$. Formally, for $C \subset A$ :

$$
\lambda_{t+1}(C)=\lambda_{t}(C)(1-\theta)+\lambda_{t}\left(A^{c}\right)(1-\alpha) \mu(C)
$$

The invariant measure of wage is now given by:

$$
\lambda(C)=\frac{(1-\alpha) \mu(C)}{\theta+(1-\alpha) \mu(A)}
$$

A second invariant probability distribution in $\left([0, D], \mathcal{B}_{[0, D]}\right)$ is given by the observed distribution of wages (rather than that of wage offers, $\lambda$ ). Call it $\lambda^{*}$.

Since all offers in $C \subset A^{c}$ are not accepted by the worker, all $\lambda$-mass in $C \subset A^{c}$ has a $\lambda^{*}$ mass concentrated at the point $w=0$. On the other hand, for sets $C \subset A, \lambda(C)=\lambda^{*}(C)$. In other words, the observed distribution of wages $\lambda^{*}$ is a mixed distribution in $[0, \bar{w}]$, with:

\footnotetext{
${ }^{5}$ It is easy to show that the adjoint operator (defined in the linear space of signed measures endowed with the total variation norm) defined by the transition function above has one and only one unique fixed point. This fact allows us to take limits in (11). This fixed point corresponds to the invariant measure of wage offers $\lambda$, defined in $\left([0, D], \mathcal{B}_{[0, D]}\right)$, which is the invariant measure we are looking for.
} 


$$
\lambda^{*}(w)= \begin{cases}\frac{\theta}{\theta+(1-\alpha) \mu(A)} & \text { if } w=0 \\ 0 & \text { if } 0<w<w^{*} \\ \frac{(1-\alpha) d F(w)}{\theta+(1-\alpha) \mu(A)} & \text { if } w \in A\end{cases}
$$

By definition, the average utility in this economy is equal to:

$$
w_{A}=\beta \int_{[0, \bar{w}]} U(w) d \lambda^{*}(w)
$$

Or, given (15), and since $U(0)=0$ :

$$
U_{A}(\beta)=\int_{\left[w^{*}, \bar{w}\right]} \frac{(1-\alpha) U(w) d F(w)}{\theta+(1-\alpha) \mu(A)}
$$

Above, $w^{*}$ is given by (7).

In the same way, the average wage $w_{A}$ of a certain worker (or of all workers in the economy at a point in time) reads: ${ }^{6}$

$$
w_{A}=\int_{\left[w^{*}, \bar{w}\right]} \frac{(1-\alpha) w d F(w)}{\theta+(1-\alpha) \mu(A)}
$$

\section{MAIN RESULTS}

Note that it is not clear from (16) and (17), at first glance, if the average utility and the average wage are increasing functions of the time-preference parameter or not. Indeed, as $\beta$ increases, so does the reservation wage, leading to a fall of $\mu(A)$. At the same time, though, the region of integration in (16) decreases. The same applies for the average wage in (17).

Even though my main focus here is on the average income, I start the calculations with the average utility solely because the results are nested: the result for the average income follows trivially from the result for the average utility under the assumption of risk neutrality.

Proposition 5.1 proves, for the case in which $F(w)$ is absolutely continuous, ${ }^{7}$ that the long-run average utility increases with patience.

Assumption 1: ${ }^{8} F^{\prime}(w)$ exists and is absolutely continuous a.e. in $[0, B]$, with $F^{\prime}(w) \equiv f(w)>0$ for $(\mu-$ a.e) all $w$ in $[0, \bar{w}]$.

${ }^{6}$ The assertion in footnote 4 should be clear by now. The cross sectional average wage of employed workers $\left(w_{A}^{E}\right)$, by the definition of conditional expectation, is equal to:

$$
E(w \mid w \in A)=\frac{1}{\lambda^{*}(A)} \int_{\left[w^{*}, \bar{w}\right]} w d \lambda^{*}(w)
$$

Using (12) and (17), we get

$$
w_{A}^{E}=\int_{\left[w^{*}, \bar{w}\right]} \frac{w d \mu(w)}{\mu(A)}
$$

as previously stated.

${ }^{7}$ The working-paper version of this article (Cysne (2004)) uses the Glivenko-Cantelli theorem (see Ferguson (1996)) to present another proof that the average wage is an increasing function of patience in a case in which $\theta=0$ and $F$ is not uniformly continuous.

${ }^{8}$ Remember the definition of $F(w)$ as the distribution function determined by $\mu$. 
Proposition 5.1. Under Assumption 1, the average utility is an increasing function of the time preference parameter $\beta$.

Proof. Using the distribution function $F$ and its respective density function $(f(w))$ with respect to the Lebesgue measure in $\mathbb{R}$, it follows from the result above that the average wage is now given by:

$$
U_{A}(\beta)=\frac{(1-\alpha)}{\theta+(1-\alpha)\left(1-F\left(w^{*}\right)\right)} \int_{\left[w^{*}, \bar{w}\right]} U(w) f(w) d w
$$

Taking the derivative in (18) with respect to $w^{*}$ :

$$
\frac{\partial U_{A}}{\partial w^{*}}=\frac{f\left(w^{*}\right)(1-\alpha)\left[(1-\alpha) \int_{\left[w^{*}, \bar{w}\right]}\left(U(w)-U\left(w^{*}\right)\right) f(w) d w-\theta U\left(w^{*}\right)\right]}{\left(\theta+(1-\alpha)\left(1-F\left(w^{*}\right)\right)^{2}\right.}
$$

The expression of the reservation wage given by (7) allows us to write:

$$
\frac{\partial U_{A}}{\partial w^{*}}=\frac{f\left(w^{*}\right)(1-\alpha) U\left(w^{*}\right)(1-\beta)}{\beta\left[\theta+(1-\alpha)\left(1-F\left(w^{*}\right)\right)\right]^{2}}>0
$$

By the chain rule, $\frac{\partial U_{A}}{\partial \beta}=\frac{\partial U_{A}}{\partial w^{*}} \frac{\partial w^{*}}{\partial \beta}$. The final expression for $\frac{\partial U_{A}}{\partial \beta}$ can therefore by obtained using (19) and (8). In particular, since both $\frac{\partial U_{A}}{\partial w^{*}}$ and $\frac{\partial w^{*}}{\partial \beta}$ have been shown to be strictly positive, so is $\frac{\partial U_{A}}{\partial \beta}$.

One could argue if this result could not be obtained by observing that, in the determination of the reservation wage, the worker would be acting suboptimally if he ended up with a lower utility on average. However, this claim is not correct. Optimality of the worker in this problem is defined in terms of the discounted value of his utility, not in terms of its long-run average utility. And a strategy to maximize $E\left(\sum_{t=0}^{\infty} \beta^{t} U\left(c_{t}\right)\right)$ is not necessarily a strategy to maximize $\lim _{T \rightarrow \infty} \frac{1}{T} \sum_{t=0}^{\infty} U\left(c_{t}\right)$. Put in another way, suboptimality in terms of $\lim _{T \rightarrow \infty} \frac{1}{T} \sum_{t=0}^{\infty} U\left(c_{t}\right)$ does not imply suboptimality in terms of $E\left(\sum_{t=0}^{\infty} \beta^{t} U\left(c_{t}\right)\right)$. The counter-example below illustrates the former assertion.

Example 5.1. Think of the limit case in which $\beta=\alpha=\theta=0$. Maximization of $E\left(\sum_{t=0}^{\infty} \beta^{t} U\left(c_{t}\right)\right)$ in this case implies choosing a reservation wage equal to zero. The worker will receive a first offer (call it $w_{0}$ ), will necessarily accept it, and will remain employed with this wage forever (since $\theta=0)$. As a result, the optimum value of $E\left(\sum_{t=0}^{\infty} \beta^{t} U\left(c_{t}\right)\right)$ is $E(U(w))$ and $\lim _{T \rightarrow \infty} \frac{1}{T} \sum_{t=0}^{\infty} U\left(c_{t}\right)=U\left(w_{0}\right)$. However, this strategy is clearly suboptimal in terms of maximizing $\lim _{T \rightarrow \infty} \frac{1}{T} \sum_{t=0}^{\infty} U\left(\left(c_{t}\right)\right)$. Indeed, since the maximum among $N$ wage offers, as $N$ tends towards infinity, converges in probability to the upper bound of the distribution $(\bar{w})$, the worker could do much better by, instead of choosing a reservation wage equal to zero, making it equal to $\bar{w}-\varepsilon$, with $\varepsilon$ positive and close enough to zero (such that $\bar{w}-\varepsilon>w_{0}$ ). By the convergence of the $n^{\text {th }}$ order statistics to $\bar{w}$, this offer would eventually be made to the worker in finite time, and he could enjoy this wage forever (since $\theta=0$ ), thereby making his average utility converge to $U(\bar{w}-\varepsilon)>U\left(w_{0}\right)$.

In sum, higher average utility here is not a synonym for higher welfare. It is, though, a synonym for higher average wages in the case of linear utility.

Assumption 2: Utility is linear $(U(w)=w)$.

Proposition 5.2. Under assumptions 1 and 2, the average wage is an increasing function of patience.

Proof. This follows trivially from Proposition 5.1.

Remark 5.1. The assumption of risk neutrality is not unusual in the job-search literature. Examples of well known papers in the area making use of this assumption are Ljungqvist and Sargent (1998), McCall (1994) and Kahn and Low (1982). 
Remark 5.2. Proposition 5.2 is the main result of this paper. It provides a new theoretical explanation for the findings that patience correlates positively with average income. ${ }^{9}$ Although the more patient worker rejects a higher number of offers, remaining more time unemployed, Proposition 5 shows that he or she ends up with a higher average wage.

Proposition 5.3. Under assumption 1, but not 2, the average wage is an increasing function of patience, provided that the probability of unemployment $\theta$ is close enough to zero.

Proof. Suffices noticing that:

$$
\frac{f\left(w^{*}\right) \int_{\left[w^{*}, \bar{w}\right]}\left(w-w^{*}\right) f(w) d w}{\left(1-F\left(w^{*}\right)\right)^{2}}>0
$$

and that:

$$
\frac{\partial w_{A}}{\partial w^{*}}=\frac{f\left(w^{*}\right)(1-\alpha)\left[(1-\alpha) \int_{\left[w^{*}, \bar{w}\right]}\left(w-w^{*}\right) f(w) d w-\theta w^{*}\right]}{\left(\theta+(1-\alpha)\left(1-F\left(w^{*}\right)\right)\right)^{2}}
$$

is a continuous function of $\theta$ for all $\theta \in[0,1)$.

Example 2 illustrates the main points of this work.

Example 5.2. Consider an economy with linear utility in which, each period, workers face a probability $\theta$ of layoff and a probability $\alpha$ of having no wage offer next period. Suppose that $\mu$ is given by the Lebesgue measure (uniform distribution) in $[0,1]$. Using (7):

$$
w^{*}(\beta)=\left[\frac{1-\beta(\alpha-\theta)}{\beta(1-\alpha)}-\sqrt{\left(\frac{1-\beta(\alpha-\theta)}{\beta(1-\alpha)}\right)^{2}-1}\right]
$$

By (17), the average wage reads:

$$
w_{A}\left(w^{*}(\beta)\right)=\frac{(1-\alpha)\left(1-w^{* 2}\right)}{2\left[\theta+(1-\alpha)\left(1-w^{*}\right)\right]}
$$

Obviously, from (20), $0<w^{*}$. Make $z(\beta)=\frac{1-\beta(\alpha-\theta)}{\beta(1-\alpha)}$. Note that $0<z<1, w^{*}(z)=z-\sqrt{z^{2}-1}$, $w^{*}(1)=1$ and that $\left(w^{*}\right)^{\prime}(z)<0$ for all $z>1$. By the mean-value theorem, this implies $w^{*}<1$. By the chain rule, the fact that $z^{\prime}(\beta)<0$ implies $\left(w^{*}\right)^{\prime}(\beta)>0$.

To show that $w_{A}^{\prime}\left(w^{*}(\beta)\right)>0$ suffices, by the chain rule, showing that $w_{A}^{\prime}\left(w^{*}\right)>0$, at the value of $w^{*}$ endogenously determined by $\beta, \alpha$, and $\theta$.

Taking the derivative in (21) with respect to $w^{*}$ leads to:

$$
w_{A}^{\prime}\left(w^{*}\right)=\frac{(1-\alpha)^{2}\left[w^{* 2}-\frac{2(\theta+1-\alpha)}{1-\alpha} w^{*}+1\right]}{2\left[\theta+(1-\alpha)\left(1-w^{*}\right)\right]^{2}}
$$

Make $g(x)=x^{2}-\frac{2(\theta+1-\alpha)}{1-\alpha} x+1$. Note that $w_{A}^{\prime}\left(w^{*}\right)>0$ if $g\left(w^{*}\right)>0$. But $g(x)$ has only one root between zero and one, which is given by:

\footnotetext{
${ }^{9}$ A subtle difference between my findings here and those of Lawarance (1991) is that I calculate $E(w \mid \beta)$, whereas Lawrance actually measures $E(\beta \mid w)$. In both cases, though, what is ultimatelly characterized in the data is a positive correlation between patience and average wage. I am thankful to one of the referees for having pointed that out to me.
} 


$$
x_{1}=\frac{\theta+1-\alpha}{1-\alpha}-\sqrt{\left(\frac{\theta+1-\alpha}{1-\alpha}\right)^{2}-1}
$$

Since $g^{\prime \prime}(x)=2>0$, all we have to prove is that $w^{*}<x_{1}$, where $x_{1}$ is given by (22). This can be done with the help of the function $h(z)$ defined above.

Since $h^{\prime}(z)<0$, if we can show that $\frac{1-\beta(\alpha-\theta)}{\beta(1-\alpha)}>\frac{\theta+1-\alpha}{1-\alpha}$, then $w^{*}<x_{1}$. This is true because $\frac{1-\beta(\alpha-\theta)}{\beta(1-\alpha)}-\frac{\theta+1-\alpha}{1-\alpha}=\frac{1-\beta}{\beta(1-\alpha)}>0$. We have proved that the average wage is an increasing function of patience.

The reservation wage and the long-run average wage for this example are illustrated in Figure 5.2, below, first as a function of beta and theta, with $\alpha=0$, and then as a function of beta and alpha, with $\theta=1 / 60$. This implies, in average, one layoff each five years, a reasonable probability of layoff.

If theta is very close to zero, the reservation wage tends to one when $\beta$ tends to one. Intuitively, the worker with beta close to one will reject a very high percentage of all offers, remaining with a wage equal to zero, whereas the worker with lower beta might accept and go on keeping his average away from zero. With theta very close to zero, though, once the patient worker accepts an offer, he remains employed with that wage for a very long period of time, eventually catching up with the less patient worker and ending up, in the long-run, with a higher average.

When either alpha or theta increase, both the reservation wage and the average wage decrease. The two graphs in the bottom show that the condition of $\theta$ being close enough to zero estabilished by Proposition 5.3 is sufficient, but by no means necessary for the average wage to increase with patience.

We can also notice that the responses of both the reservation wage and the average wage to patience tend to decrease when either the probability of lay off or the probability that unemployed workers do not get a job offer increases. This can be easily seen by focusing on the level lines parallel to the axis where beta is measured, both for low and high and values of theta and alpha, respectively.

\section{CONCLUSIONS}

This paper has used a standard job-search model to provide a new explanation for the empirically assessed positive correlation between patience and average income: more patient workers, by rejecting less favorable wage offers, end up with a higher income in the long run. The point is not clear at first sight, since rejecting more offers also leads to more time of unemployment. However, assuming linear utility, the paper shows that more patient workers always "win in the end", meaning that they end up with a higher average income. When the utility is not linear, the result is also true, provided that the probability of unemployment is small enough.

The average utility of the more patient worker was also proved to increase with patience. In this case, no additional assumption is necessary.

A counter example was used to show that the conclusions of the paper could not follow from a direct analysis of the value function, and that higher average utility here is not a synonym for higher welfare.

A second example at the end of the text was used to illustrate how the positive influence of patience on average income depends on the probability of unemployment and on the probability that unemployed workers do not receive a job offer.

\section{Bibliography}

Andolfatto, D. \& Redekop, J. (1998). Heterogeneous time preference and the distribution of wealth. Economics Department, University of Waterloo. 
Becker, R. A. (1980). On the long-run steady state in a simple dynamic model of equilibrium with heterogeneous households. Quarterly Journal of Economics, 95(2):375-82.

Carroll, C. (1997). Buffer-stock saving and the life cycle/permanent income hypothesis. Quarterly Journal of Economics, 112(1):1-55.

Cysne, R. P. (2004). On the negative correlation between labor income and impatience. Technical Report 561, Escola de Pós-Graduação em Economia da FGV. Ensaio Econômico da EPGE.

Dreyfus, M. K. \& Viscusi, W. K. (1995). Rates of time preference and consumer valuations of automobile safety and fuel efficiency. Journal of Law and Economics, 38:79-105.

Ferguson, T. S. (1996). A Course in Large Sample Theory. Chapman\&Hall / CRC.

Hausman, J. A. (1979). Individual discount rates and the purchase and utilization of energy-using durables. Bell Journal of Economics, 10(1):33-54.

Kahn, M. L. \& Low, S. A. (1982). The relative effects of employed and unemployed job search. The Review of Economics and Statistics, 64(2):234-241.

Krusell, P. \& Smith, A. (1998). Income and wealth heterogeneity in the macroeconomy. Journal of Political Economy, 106(5):867-96.

Lawarance, C. E. (1991). Poverty and the rate of time preference: Evidence from panel data. The Journal of Political Economy, 99(1):54-77.

Ljungqvist, L. \& Sargent, T. J. (1998). The european unemployment dilemma. The Journal of Political Economy, 106(3):514-550.

Lucas, E. R. \& Stokey, N. (1984). Optimal growth with many consumers. Journal of Economic Theory, pages 139-171.

MacCall, J. J. (1970). Economics of information and job search. Quarterly Journal of Economics, 84:113126.

Maital, S. \& Maital, S. (1977). Time Preference, Delay Gratification and the Intergenerational Transmission of Economic Inequality: A Behavioral Theory of Income Distribution. in Essays in Labor Market Analysis in Memory of Yochanan Peter Comay.

McCall, P. B. (1994). he effect of job heterogeneity on reservation wages. International Economic Review, 35(3):773-791.

Samwick, A. A. (1998). Discount rate heterogeneity and social security reform. Journal of Development Economics, 57:117-146.

Sargent, T. (1987). Dynamic Macroeconomic Theory. Harvard University Press.

Sargent, T. \& Ljungqvist, L. (2002). Recursive Macroeconomic Theory. MIT Pres.

Stokey, N. L., Lucas, R., \& Prescott, E. C. (1989). Recursive Methods in Economic Dynamics. Harvard University Press.

Warner, J. T. \& Pleeter, S. (2001). The personal discount rate: Evidence from military downsizing programs. American Economic Review, 91(1):33-53.

Wärneryd, K. E. (1999). The Psychology of Saving: A Study on Economic Psychology. Edward Elgar. 
R.W. (Theta, Beta), (Alpha=0)

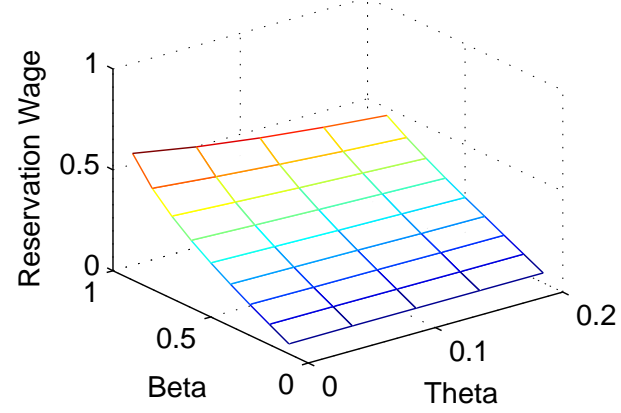

A. W. (Theta, Beta), (Alpha=0)

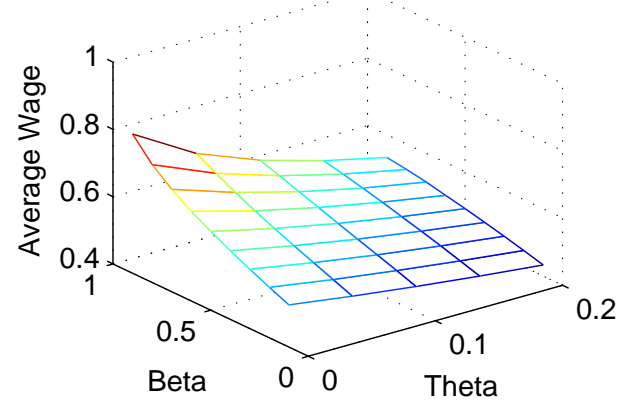

R.W. (Alpha, Beta), (Theta $=1 / 60)$

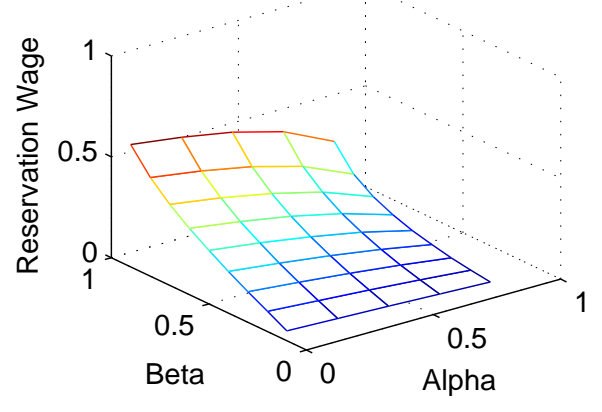

A. W. (Alpha, Beta), (Theta $=1 / 60)$

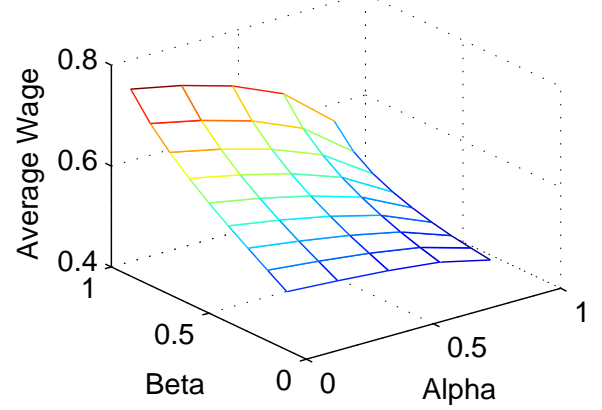

